\title{
Suppressing Weak Ising Couplings: Tailored Gates for Quantum Computation
}

\author{
Jonathan A. Jones \\ Centre for Quantum Computation, Clarendon Laboratory, Parks Road, Oxford \\ OX1 3PU, United Kingdom.
}

\begin{abstract}
I describe the use of techniques based on composite rotations to develop controlled phase gates in which the effects of weak Ising couplings are suppressed. A tailored composite phase gate is described which both suppresses weak couplings and is relatively insensitive to systematic errors in the size of strong couplings.
\end{abstract}

Key words: Quantum computation, Ising coupling, nuclear magnetic resonance PACS: 03.67.Lx, 76.60.-k

Quantum computers [1] are extremely vulnerable to the effects of errors, and there has been considerable interest in correcting random errors arising from decoherence processes $[2,3,4]$ and, more recently, in stabilising logic gates against systematic errors, which arise from reproducible imperfections in the system used to implement quantum computations. In the context of Nuclear Magnetic Resonance (NMR) quantum computation $[5,6,7,8]$ systematic errors can be reduced using composite rotations (composite pulses) $[9,10,11,12]$, which have been applied to both single qubit gates $[13,14]$ and the two qubit controlled phase-shift gate $[15,16]$.

Here I consider a related but distinct problem: the design of composite phase gates which automatically suppress evolution under small Ising couplings. This is useful, as many proposed implementations involve extended networks of Ising couplings. Typically in such systems the nearest neighbour interactions are large, and form the basis of two qubit gates, while next-nearest neighbour interactions are much weaker. Frequently such interactions are too weak to be useful, but too strong to simply be ignored: it is, therefore, useful to develop sequences which are capable of distinguishing between small and large cou-

$\bar{*}$ Email address: jonathan.jones@qubit.org 
plings, effectively suppressing small couplings. Ideally such sequences should also be robust to small errors in the size of the large couplings.

As described previously $[15,16]$ it is straightforward to convert a robust single qubit gate into a robust controlled phase-shift gate; as single qubit gates are much easier to study it makes sense to adopt this approach. A composite rotation comprises a series of rotations applied in sequence, such that the overall effect in the absence of errors is the desired rotation, while in the presence of systematic errors the effect of these errors largely cancels out. With some approaches to designing composite rotations it can be difficult to ensure that both requirements are simultaneously true, but this problem can be side-stepped using a method due to Wimperis [12], in which the composite rotation is constructed by combining a simple rotation with a complex series of additional rotations, which in the absence of errors does nothing. This approach guarantees that the composite rotation will behave correctly in the absence of errors, while allowing error tolerance to be developed by tweaking the do-nothing sequence. Note that although these composite rotations are developed in the context of NMR they are applicable to other implementations of quantum computation based on Ising couplings [17,18,19,20,21].

Single qubit gates are transformations in $\mathrm{SU}(2)$, and so can be conveniently represented by quaternions [14]. The composite quaternion for a composite rotation can be obtained by multiplying the quaternions for the individual rotations, and then expanding the result as a Maclaurin series in the fractional error. As an example consider the BB1 sequence [12,14] for reducing the effects of errors in rotation rates (traditionally called pulse length errors in NMR): the simple pulse $\theta_{x}$ is replaced by the composite pulse sequence

$$
(\theta / 2)_{x} 180_{\phi_{1}} 360_{\phi_{2}} 180_{\phi_{1}}(\theta / 2)_{x}
$$

where the phase angles $\phi_{1}$ and $\phi_{2}$ are chosen to make the rotation error tolerant. If the rotation errors are parameterised using the fractional error in the rotation rate, $g$, then the simple rotation shows first order errors in $g$, but these can be removed by choosing $\phi_{2}=3 \phi_{1}$ and

$$
\phi_{1}= \pm \arccos \left(-\frac{\theta}{4 \pi}\right) \text {. }
$$

Remarkably this approach also removes the errors which are second order in $g$.

The BB1 approach can easily be adapted to tackle the new problem: developing a composite rotation which has only minimal effect when the rotation rate is very small. (When converted to its two qubit equivalent, this will create a controlled phase-shift gate which effectively suppresses evolution under small Ising couplings). This can be achieved by comparing the composite quaternion 
(a)

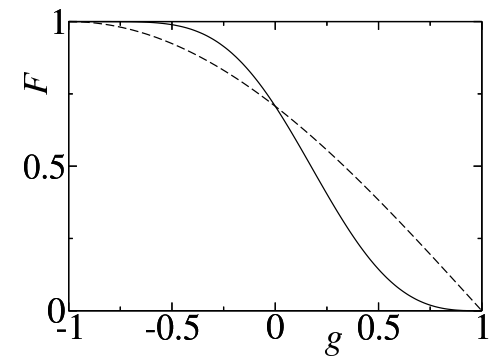

(b)

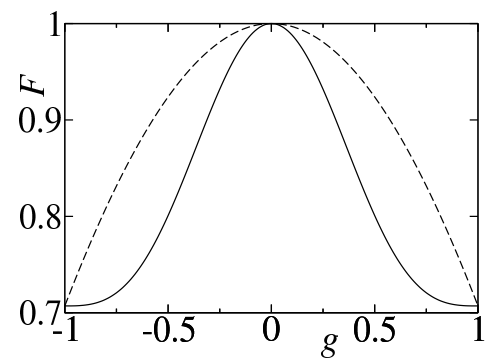

Fig. 1. Fidelity $F$ of simple (dashed line) and selective NB1 (solid line) $90^{\circ}$ rotations as a function of the fractional error in the rotation rate $g$. Fidelities are measured (a) against an ideal $0^{\circ}$ rotation (the identity operation), and (b) against a $90^{\circ}$ rotation. An ideal composite rotation would show broad plateaus of height 1 at $g=-1$ in plot (a) and at $g=0$ in plot (b).

with the null quaternion

$$
\mathrm{q}_{0}=\{1,\{0,0,0\}\}
$$

and then replacing the Maclaurin series expansion with a Taylor series expansion around the point $g=-1$. As observed previously [16], the $z$-component of the composite quaternion is automatically correct, while the first order $y$ component can be removed by choosing $\phi_{2}=-\phi_{1}$. Finally $\phi_{1}$ must be chosen to zero the first order $x$-component, and is once again given by Eq. 2 .

The results of this composite rotation (which will be called NB1 for reasons that will become clear later) for the case $\theta=90^{\circ}$ are shown in Fig. 1. This shows the fidelity $F$ of simple and composite rotations as a function of the fractional error in the rotation rate $g$. Note that for single qubit gates the propagator and quaternion fidelities are the same $[14,15,16]$. An ideal composite rotation would show broad plateaus of height 1 at $g=-1$ in plot (a), indicating effective suppression of small couplings, and at $g=0$ in plot (b), indicating robust evolution under large couplings. Clearly the NB1 composite rotation is effective at suppressing evolution around $g=-1$, but it achieves this at the expense of reducing the robustness of the sequence in the low-error region, around $g=0$. This is not particularly surprising, and becomes even less so on the discovery that this composite rotation is in fact essentially identical to the first narrowband excitation family (NB1) described by Wimperis [12]. (The only difference is that the new version has been time symmetrised, by placing the correction pulses half way through the main pulse, rather than at the start).

As two interesting composite rotations (BB1 and NB1) are in fact rediscoveries of results by Wimperis, it seems sensible to examine his other results to see what else might be found. He describes [12] two other families of broadband and narrow band pulses, BB2 and NB2, but these are not suitable for quantum computing. He also describes two families of "passband" composite rotations, PB1 and PB2, and remarkably PB1 is exactly the sequence we are seeking (PB2 is not suitable for quantum computing). The time symmetrised version 
(a)

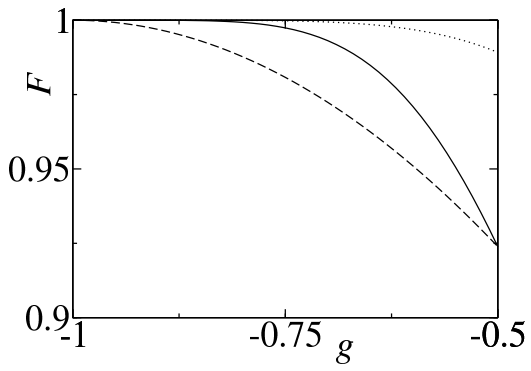

(b)

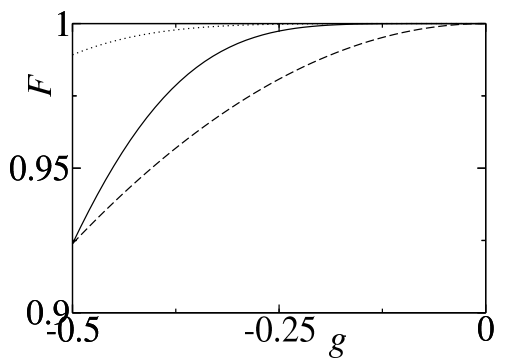

Fig. 2. Fidelity of simple (dashed line), PB1 (solid line), and NB1 or BB1 (dotted line) $90^{\circ}$ rotations as a function of the fractional error in the rotation rate $g$ : (a) fidelity measured against an ideal $0^{\circ}$ rotation (the identity operation), with the dotted line showing the NB1 sequence; (b) fidelity measured against a $90^{\circ}$ rotation, with the dotted line showing the BB1 sequence. Note that the horizontal axes differ in the two plots.

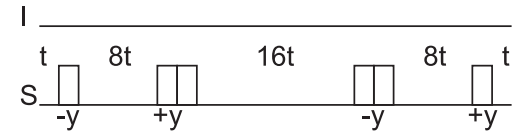

Fig. 3. Pulse sequence for an Ising gate to implement a controlled-NOT gate which both suppresses small couplings and is robust to small errors in coupling sterngths. Boxes correspond to single qubit rotations with rotation angles of $\phi=\arccos (-1 / 16) \approx 93.6^{\circ}$ applied along the $\pm y$ axes as indicated; time periods correspond to free evolution under the Ising coupling, $\pi J 2 I_{z} S_{z}$ for multiples of the time $t=1 / 4 J$. The naive Ising gate corresponds to free evolution for a time $2 t$.

takes the form

$$
(\theta / 2)_{x} 360_{\phi_{1}} 720_{\phi_{2}} 360_{\phi_{1}}(\theta / 2)_{x}
$$

with $\phi_{2}=-\phi_{1}$ and

$$
\phi_{1}= \pm \arccos \left(-\frac{\theta}{8 \pi}\right) .
$$

The performance of the PB1 sequence is shown in Fig. 2. This sequence is in some sense a compromise between NB1 and BB1: it performs worse than NB1 near $g=0$ and worse than BB1 near $g=-1$, but outperforms a simple pulse in both domains, exactly as desired.

Finally I return to the problem of converting a single qubit PB1 composite rotation into the desired two qubit controlled phase-shift gate, which both suppresses small couplings and is robust to small errors in coupling strengths. The controlled phase-shift gate is equivalent to the Ising coupling gate [8], and a robust Ising gate can be built as described previously [15]. After combining and cancelling extraneous pulses, the final sequence for the case $\theta=\pi / 2$ (which forms the basis of the controlled-NOT gate) is shown in Fig. 3. For two qubit gates the natural definition of the fidelity is given by the propagator fidelity [15], but for two qubit gates of this kind, which are essentially equivalent to single qubit gates, the propagator fidelity is once again equivalent to the quaternion fidelity [16]. 
Following previous work [15], I assume that an infidelity $(1-F)$ of $10^{-6}$ can be tolerated in a quantum logic gate. A useful measure of the practicality of an Ising coupling gate is then provided by the maximum fractional error $\epsilon$ in the coupling constant $J$ before this tolerance is exceeded [16]. Another useful measure, indicating the ability of the gate to suppress small couplings, is the largest fractional coupling $\delta$ which cannot simply be neglected. On these measures the simple Ising gate does not perform well: it requires that $J$ be controlled to better than $0.2 \%(\epsilon<0.0018)$ and the limit on small couplings is the same $(\delta<0.0018)$. The BB1 gate corrects well for small errors in $J$, permitting errors of up to $10 \%(\epsilon<0.1015)$, but is even more sensitive to small couplings, requiring $\delta<0.0009$. As might be guessed from the symmetry of the two plots in Fig. 2 the performance of NB1 is exactly the opposite, allowing small couplings to be effectively suppressed $(\delta<0.1015)$, but at the cost of increased sensitivity to errors $(\epsilon<0.0009)$. Finally the PB1 sequence is a compromise between NB1 and BB1, with $\delta<0.0648$ and $\epsilon<0.0648$.

For most purposes the PB1 sequence provides the most practical Ising gate currently known: at an infidelity of $10^{-6}$ it can tolerate errors of more than $6 \%$ in coupling constants, but it also permits small couplings, with strengths up to $6 \%$ of the coupling being used to implement a gate, to be neglected. If even higher fidelities are required then these bounds will of course be reduced, but the relative improvement provided by the PB1 sequence will be even greater.

I thank S. Benjamin for helpful conversations and the UK EPSRC for financial support.

\section{References}

[1] C. H. Bennett, and D. P. DiVincenzo, Nature (London) 404, 247 (2000).

[2] P. W. Shor, Phys. Rev. A 52, 2493 (1995).

[3] A. Steane, Phys. Rev. Lett. 77, 793 (1996).

[4] A. Steane Nature 399, 124 (1999).

[5] D. G. Cory, A. F. Fahmy and T. F. Havel, in Proceedings of PhysComp '96 (New England Complex Systems Institute, Cambridge MA, 1996).

[6] D. G. Cory, A. F. Fahmy and T. F. Havel, Proc. Nat. Acad. Sci. USA 94, 1634 (1997).

[7] N. A. Gershenfeld and I. L. Chuang, Science 275, 350 (1997).

[8] J. A. Jones, Prog. NMR. Spectrosc. 38, 325 (2001).

[9] R. R. Ernst, G. Bodenhausen, and A. Wokaun, Principles of Nuclear Magnetic Resonance in One and Two Dimensions (Oxford University Press, 1987). 
[10] R. Freeman, Spin Choreography (Spektrum, Oxford, 1997).

[11] M. H. Levitt, Prog. NMR Spectrosc. 18, 61 (1986).

[12] S. Wimperis, J. Magn. Reson. A 109, 221 (1994).

[13] H. K. Cummins and J. A. Jones, New J. Phys. 2, 1 (2000).

[14] H. K. Cummins, G. Llewellyn and J. A. Jones, Phys. Rev. A. 67, 042308 (2003).

[15] J. A. Jones, Phys. Rev. A 67, 012317 (2003).

[16] J. A. Jones, Phil. Trans. R. Soc. Lond. A 361, 1429 (2003).

[17] S. Lloyd, Science 261, 1569 (1993).

[18] L. B. Ioffe, V. B. Geshkenbein, M. V. Feigel'man, A. L. Fauchère, and G. Blatter, Nature 398, 679 (1999).

[19] J. I. Cirac and P. Zoller, Nature 404, 579 (2000).

[20] H. J. Briegel and R. Raussendorf, Phys. Rev. Lett. 86, 910 (2001).

[21] R. Raussendorf and H. J. Briegel, Phys. Rev. Lett. 6, 5188 (2001). 